\title{
Diagnóstico clínico-radiológico de neurocisticercosis: a propósito de un caso
}

\section{Clinical and radiological diagnosis of neurocysticercosis: A case report}

\author{
D. Navarro' ${ }^{1}$ I. Huarte ${ }^{1}$, R. Santesteban ${ }^{1}$, M. Bidarte ${ }^{1}$, A. Ayechu ${ }^{2}$
}

\section{RESUMEN}

Introducción. La neurocisticercosis es la enfermedad parasitaria más frecuente del sistema nervioso central y la primera causa de epilepsia de inicio tardío en las áreas endémicas. Nuestro objetivo con este trabajo es orientar a través de los criterios clínicos y radiológicos el diagnóstico de sospecha de esta enfermedad, presentando un caso clínico.

Materiales y métodos. Presentamos el caso de una mujer de 43 años, natural de Bolivia, que acudió al Servicio de Urgencias tras presentar una crisis convulsiva generalizada, presenciada por familiares de la paciente.

Resultados. Se realizó una tomografía axial computarizada craneal, que permitió junto con su historia clínica sospechar una neurocisticercosis. Se ingresó en neurocirugía para completar el estudio, que confirmó el diagnóstico de sospecha, y recibió tratamiento con albendazol y corticoides, con buena evolución.

Conclusiones. La neurocisticercosis es una patología emergente en países desarrollados, debido al aumento de la inmigración desde áreas endémicas, principalmente de América Latina. La epilepsia es la expresión clínica más frecuente, pero la presentación es muy variable. Un alto grado de sospecha es necesario para poder diagnosticar esta enfermedad.

Palabras clave. Neurocisticercosis. Epilepsia. Inmigrante. Albendazol. Taenia solium.

\begin{abstract}
Background. Neurocysticercosis is the most frequent parasitic disease of the central nervous system and the first cause of acquired epilepsy in endemic areas. Our aim with this paper is to use clinical and radiological criteria to orientate diagnosis where there is suspicion of neurocysticercosis, presenting a clinical case as an example.
\end{abstract}

Materials and methods. We present the case of a 43 year old woman of Bolivian origin, who came to accidents and emergencies after suffering a generalized convulsive crisis, witnessed by relatives of the patient.

Results. A cranial computerized axial tomography was taken, which together with her clinical history led to suspicion of neurocysticercosis. She was admitted to the neurosurgery department for completion of the study, which confirmed the diagnosis of suspicion. She received treatment with albendazol and corticoids, with a good evolution.

Conclusions. Neurocysticercosis is an emergent pathology in developed countries, due to the increase of immigration from endemic areas, mainly from Latin America. Epilepsy is the most frequent clinical expression, but presentation can vary greatly. A high degree of suspicion is necessary in order to be able to diagnose this disease.

Key words. Neurocysticercosis. Epilepsy. Immigrant Albendazole. Taenia solium.
1. Centro de Salud de San Jorge. Servicio Navarro de Salud-Osasunbidea. Pamplona.

2. Servicio de Pediatría. Hospital Virgen del Camino. Pamplona.

Recepción: 3 de febrero 2009

Aceptación provisional: 12 de febrero 2009

Aceptación definitiva: 17 de febrero 2009

\author{
Correspondencia \\ David Navarro González \\ Comunidad de La Rioja $1-3^{\circ} \mathrm{C}$ \\ 31010 Barañain (Navarra) \\ Tfno. 615984278 \\ Fax 948136286
}




\section{INTRODUCCIÓN}

La neurocisticercosis (NCC) es causada por la forma larvaria (cisticerco) de la Taenia solium (TS) del cerdo. La cisticercosis se adquiere cuando el hombre ingiere los huevos por alimentos-agua contaminados, por contacto con otras personas o se infestan por auto-contaminación. Cuando las personas ingieren carne de cerdo mal cocida con cisticercos viables, estas larvas se evaginan en el intestino delgado, el escólex se adhiere a la pared intestinal y el cuerpo del parásito comienza a crecer, provocando una inflamación en la zona de implantación, la teniasis intestinal. Cada día expulsa varios proglótides que son eliminados con las heces. En el caso de que los huevos atraviesen el intestino, se distribuyen por el torrente sanguíneo a los diferentes tejidos del hombre, con gran afinidad por el músculo y el sistema nervioso central (SNC), donde pueden provocar los síntomas típicos de la $\mathrm{NCC}^{1,2}$.

La cisticercosis era frecuente en Europa y endémica en España ${ }^{3}$ a principios de siglo $\mathrm{XX}$; sin embargo, mejoras generales en el sistema de salud pública produjeron una reducción de su prevalencia ${ }^{4,5}$. La mayoría de las personas afectas de NCC son inmigrantes provenientes de áreas endémicas, principalmente América Latina ${ }^{6,7}$, Asia y África. El aumento del turismo y la inmigración de individuos procedentes de estas áreas endémicas han condicionado un aumento en la incidencia en países desarrollados ${ }^{5,8}$.

La NCC es un problema de salud pública, considerada la enfermedad parasitaria más frecuente del SNC y la primera causa de epilepsia de inicio tardío en las áreas endémicas $^{9,10}$.

Nuestro objetivo con este trabajo es orientar a través de los criterios clínicos y radiológicos el diagnóstico de sospecha de la NCC, presentando un caso clínico como ejemplo de la experiencia en urgencias del abordaje clínico de un paciente afecto de NCC.

\section{CASO CLÍNICO}

Se trata de una mujer de 43 años, natural de Bolivia, que acudió al Servicio de Urgencias del Hospital de Navarra tras presentar en su domicilio una crisis convulsiva generalizada, presenciada por familiares, con un periodo confusional posterior y una cefalea intensa bilateral. Previo al cuadro una hora antes comenzó con parestesias en mano derecha. No tenía antecedentes familiares ni antecedentes personales de interés.
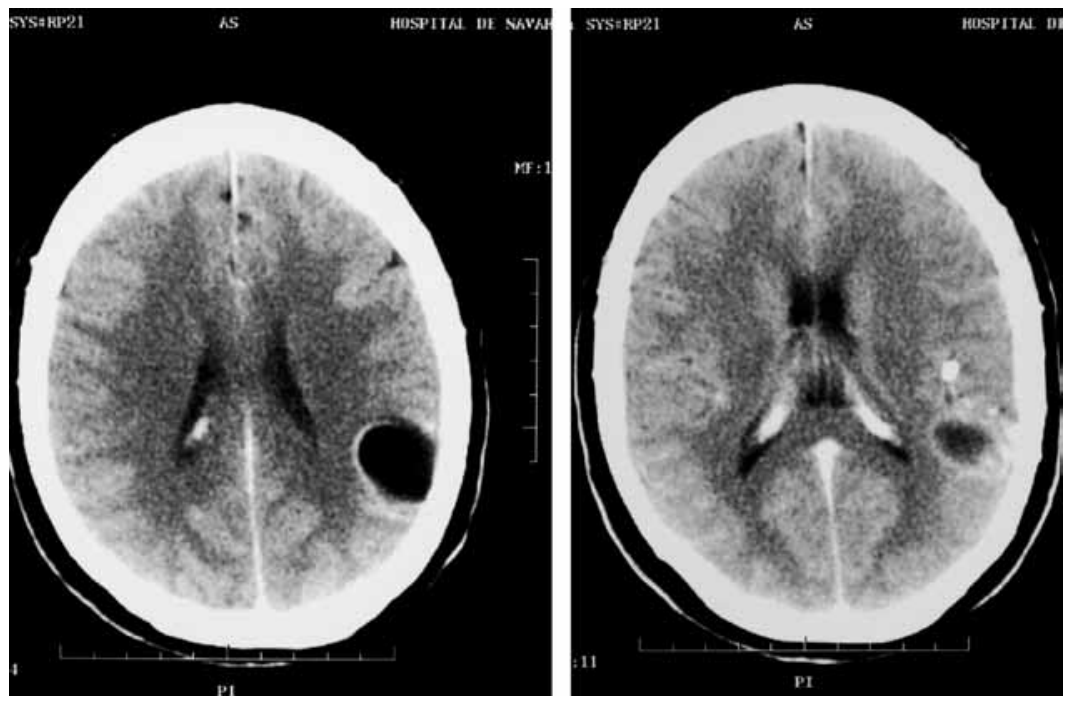

Figura 1. TAC craneal donde se observa una lesión quística de $3 \mathrm{~cm}$ de diámetro, con calcificaciones nodulares en su periferia, que se corresponde con una nuerocisticercosis. 
En la exploración física, presentaba constantes de presión, frecuencia y temperatura normales y un Glasgow de 15. No presentaba rigidez de nuca ni signos meníngeos. No se encontró focalidad neurológica. La paciente se encontraba sudorosa, con un cuadro de ansiedad asociado, que fue resuelto con éxito con $0,25 \mathrm{mg}$ de alprazolam. El resto de la exploración fue normal y no presentó nueva crisis.

En el Servicio de Urgencias se realizó ECG, radiografía de tórax, ambos normales, y analítica con bioquímica, hemograma y pruebas de coagulación, también dentro de los parámetros normales. Se practicó TAC craneal urgente (Fig. 1), donde se objetivó una lesión de $3 \mathrm{~cm}$ de diámetro, quística, en región fronto-parietal izquierda, que no captaba contraste, con calcificaciones nodula- res en su periferia. Se decidió ingreso en neurocirugía con diagnóstico de sospecha de NCC.

Durante su ingreso se completó el estudio con un electrencefalograma (EEG), que fue normal, una resonancia magnética craneal (Fig. 2A), donde se confirmó la existencia de cisticercos en fase vesicular, y una radiografía del muslo (Fig. 2B), con varias calcificaciones de pequeño tamaño y morfología compatible con cisticercos. Se inició tratamiento con dexametasona, albendazol y oxcarbazepina, con buena tolerancia, y se le dio el alta con el diagnóstico de epilepsia rolándica izquierda secundaria a cisticercosis sistémica y terminó el tratamiento ambulatorio durante un mes con albendazol, manteniendo de manera indefinida el tratamiento antiepiléptico, sin presentar nuevas crisis.

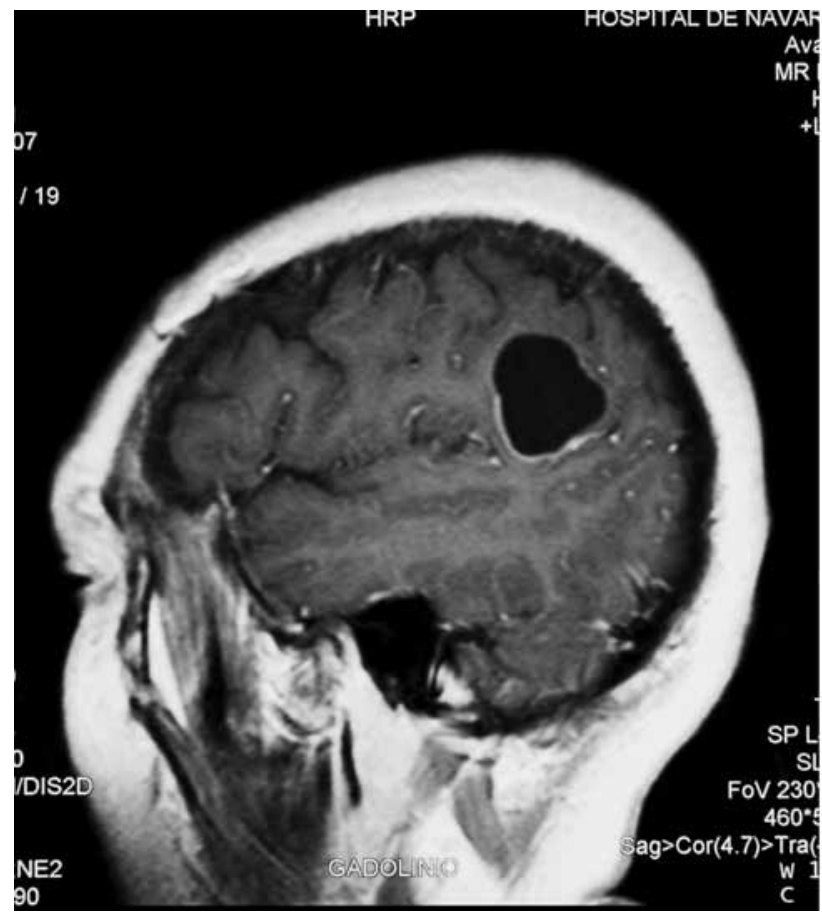

Figura 2A. Resonancia magnética craneal donde se aprecia la presencia de cisticercos en fase vesicular.

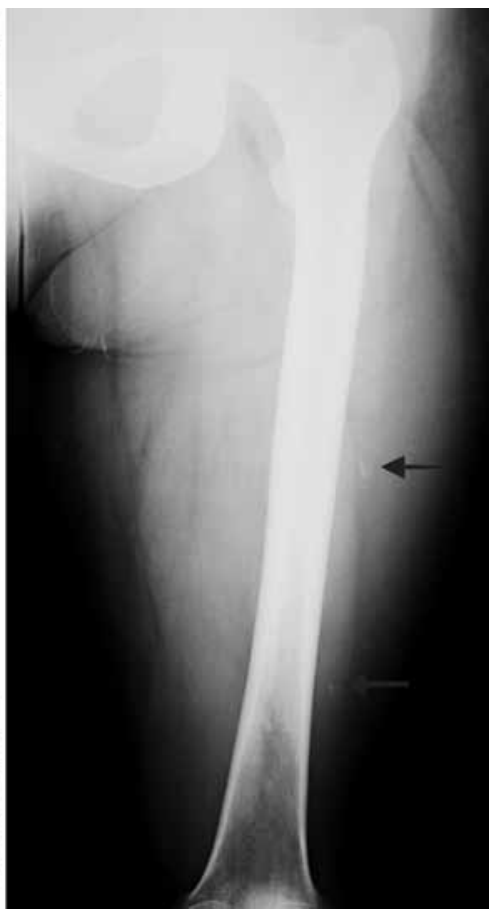

Figura 2B. Radiografía del muslo con calcificaciones.

\section{DISCUSIÓN}

La NCC es una enfermedad pleomórfica debida a las diferentes respuestas inmuni- tarias de cada huésped frente a la infección parasitaria y a la localización variable de las lesiones. La epilepsia es la clínica de aparición más frecuente $(50-80 \%)^{1}$, un ter- 
cio de ellas generalizadas, y en un $4 \%$, estatus epiléptico. No obstante, otras formas clínicas como una cefalea tipo migraña con o sin aura (muy frecuente manifestación en niños) o hemiparesias-hemiparestesias deben hacer sospechar una $\mathrm{NCC}^{11}$ en especial en pacientes procedentes de áreas endémicas. La localización de los quistes del cisticerco es predominantemente intraparenquimatosa cerebral, pero no es raro encontrar quistes en otras localizaciones como espinal, espacio subaracnoideo o extraneural. Destaca su afinidad por el tejido muscular y el tejido subcutáneo.

Para el diagnóstico correcto de la NCC es necesaria la integración de los hallazgos clínicos, neuroimagen y serológicos ${ }^{12,13}$, integrados en un correcto contexto epidemiológico. Según el grado de certeza se llega a un diagnóstico probable o definitivo (Tabla $1)^{1,11,13}$. Los hallazgos más característicos de neuroimagen en la NCC parenquimatosa son las calcificaciones y las lesiones quísti- cas, hallazgos no específicos, que también pueden observarse en otras infecciones del SNC. La presencia de cisticercosis extraneuronal facilita el diagnóstico en pacientes con clínica compatible y neuroimagen no concluyente. Existen otras pruebas como son el ELISA y el inmunoblot que permiten detectar anticuerpos anticisticerco en sangre o en LCR. Estas pruebas pueden ayudar al diagnóstico pero nunca de forma aislada para confirmarlo ${ }^{1}$. Además, pacientes con NCC calcificada pueden cursar con pruebas inmunológicas negativas. Nuestra paciente presentaba lesiones sugestivas en la TAC (criterio mayor), clínica compatible (criterio menor) y procedencia de un área endémica (criterio epidemiológico), además de cisticercosis extraneuronal en la radiografía de muslos, lo que permitió el diagnóstico probable y su ingreso para inicio del tratamiento. El tratamiento de la NCC incluye fármacos cisticidas, medidas sintomáticas y cirugía.

Tabla 1. Criterios diagnósticos de $\mathrm{NCC}^{10,11}$.

\begin{tabular}{|c|c|}
\hline \multicolumn{2}{|c|}{ Criterios diagnósticos } \\
\hline $\begin{array}{l}\text { Criterios absolutos } \\
\text { - Demostración histológica del parásito en biopsia de } \\
\text { cerebro o médula espinal. } \\
\text { - Presencia de lesiones quísticas mostrando el scólex } \\
\text { en TAC o RM. } \\
\text { - Visualización de un cisticerco subretiniano en el } \\
\quad \text { fondo del ojo. }\end{array}$ & $\begin{array}{l}\text { Criterios menores } \\
\text { - Lesiones compatibles de NCC en estudios de } \\
\text { neuroimagen. } \\
\text { - } \text { Manifestaciones clínicas sugestivas de NCC. } \\
\text { - Presencia de Ac anticisticerco en LCR mediante } \\
\text { ELISA. } \\
\text { - Presencia de cisticercos extraneuronal. }\end{array}$ \\
\hline $\begin{array}{l}\text { Criterios mayores } \\
\text { - Lesiones sugestivas de NCC en examen de } \\
\text { neuroimagen. } \\
\text { - Presencia de Ac anticisticercos en inmonoblot. } \\
\text { - Resolución de lesiones quísticas tras tratamiento } \\
\text { cesticida. } \\
\text { - Resolución espontánea de lesiones anulares únicas. }\end{array}$ & $\begin{array}{l}\text { Criterios epidemiológicos } \\
\text { - Evidencia de contacto familiar con infección por } \\
\text { Taenia solium. } \\
\text { - Sujetos que habitan en áreas endémicas. } \\
\text { - Historia de viajes a zonas endémicas. }\end{array}$ \\
\hline
\end{tabular}

Grado de certeza diagnóstica

\footnotetext{
Diagnóstico definitivo

- Un criterio absoluto

- Dos criterios mayores más un criterio menor y un epidemiológico.

\section{Diagnósticos probables}

- Un criterio mayor y dos criterios menores.

- Un criterio mayor más un criterio menor y un epidemiológico.

- Tres criterios menores y un epidemiológico.
} 
Los pacientes con quistes viables deben recibir tratamiento cisticida. El fármaco de elección por su mayor porcentaje de destrucción de quistes parenquimatosos y por su coste es albendazol, a dosis de $15 \mathrm{mg} / \mathrm{kg} /$ día durante 30 días $^{14,20}$, aunque estudios anteriores demostraron iguales resultados con 7 días de tratamiento ${ }^{15,16}$. Los pacientes con calcificaciones cuando presenten crisis convulsivas deben recibir antiepilépticos. La duración del tratamiento no está definida por el alto índice de recidivas al suspender los antiepilépticos ${ }^{17}$. El tratamiento quirúrgico se reserva a casos refractarios o en casos de compromiso neurológico grave: hipertensión intracraneal, hidrocefalia o compresión medular ${ }^{18}$.

Por último, medidas como la vacunación preventiva de cerdos con vacunas con antígenos recombinantes ${ }^{19}$, permitiría finalmente interrumpir el ciclo de vida del parásito y el control de la enfermedad en un futuro próximo.

\section{BIBLIOGRAFÍA}

1. Del Brutto OH. Neurocysticercosis: up-dating in diagnosis and treatment. Neurología 2005; 20: 412-418.

2. Imirizaldu L, Miranda L, García-Gurtubay I, GasTón I, URRIZA J, Quesada P. Neurocisticercosis. Una enfermedad emergente. An Sist Sanit Navar 2004; 27: 201-209.

3. García-Albea E. Cisticercosis en España. Algunos datos epidemiológicos. Rev Clin Esp 1989; 184: 3-6.

4. Mitchell WG, CRAWFoRd TO. Intraparenchymal cerebral cysticercosis in children: diagnosis and treatment. Pediatrics 1988; 82: 76-82.

5. Engels D, Urbani C, Belotto A, Meslin F, Savioli L. The control of human (neuro) cysticercosis: Which way forward? Acta Trop 2003; 87: 177-182.

6. Roca C, Gascón J, Font B, Pujol T, Valls ME, CoRACHÁN M. Neurocysticercosis and population movements: analysis of 23 imported cases in Spain. Eur J Clin Microbiol Infect Dis 2003; 22: 382-384.

7. Terraza S, Pujol T, Gascon J, Corachan M. Neurocysticercosis: an imported disease? Med Clin 2001; 116: 261-263.
8. JunYent M, NúÑEz S, Miró O. Urgencias médicas del inmigrante adulto. An Sist Sanit Navar 2006; 29 (Suppl 1): 27-34.

9. Whiтe A. (1997). Neuroysticercosis: a major cause of neurological disease worldwide. Clin Infect Dis 1997; 24: 101-115.

10. FERRER E. Taeniasis/cysticercosis: Epidemiology and control. Advances in vaccine production. Bol Mal Salud Amb 2005; 45: 89-99.

11. Sotelo J, Del Brutto OH. Review of neurocysticerocis. Neurourg Focus 2002; 12: 1-7.

12. García HH, Del Brutto OH, Nash TE, White aC JR, Tsang VC, Gilman RH. New concepts in the diagnosis and management of neurocysticercosis (Taenia solium). Am J Trop Med Hyg 2005; 72: 3-9.

13. Del Brutto OH, Rajshekhar V, White AC JR, Tsang VC, Nash TE, Takayanagu OM et al. Proposed diagnostic criteria for neurocysticercosis. Neurology 2001; 57: 177-183.

14. Escobedo F, Sotelo J, Penagos P, Rodriguez J, Del BRUTTO O. Albendazole therapy for human neurocysticerosis, a controlled study with computerized tomography and magnetic resonante. Acta Leiden 1989; 57: 247-254.

15. Sotelo J, Del Brutto OH, Penagos P, Escobedo F, Torres B, Rodríguez-Carbajal J et al. Comparison of therapeutic regimen of anticysticercal drugs for parenchymal brain cisticercosis. J Neurol 1990; 237: 69-72.

16. García HH, Gilman RH, Horton J, Martínez M, Herrera G, Altamirano J et al. Albendazole therapy for neurocysticercosis: a prospective double-blind trial comparing 7 vs 14 days of treatment. Neurology 1997; 48: 1421-1427.

17. Nash Te, Del Brutto OH, Butman Ja, Corona T, Delgado-Escuela A, Duron RM et al. Calcific neurocysticercosis and epileptogenesis. Neurology 2004; 62: 1934-1938.

18. Hawk MW, Shahlaie K, Kim KD, Theis JH. Neurocysticercosis: a review. Surg Neurol 2005; 63: 123-132.

19. FERRER E. Taeniasis/cysticercosis: epidemiology and control. Advances in vaccine production. Bol Mal Salud Amb 2005; 45: 89-99.

20. Pérez-López C, Isla-Guerrero A, Álvarez F, Budke M, Fernández-Miranda JC, Paz JF et al. Actualización en el tratamiento de la neurocisticercosis. Rev Neurol 2003; 36: 805-811. 
